University of Nebraska - Lincoln

DigitalCommons@University of Nebraska - Lincoln

Faculty Publications in Computer \& Electronics Electrical \& Computer Engineering, Department Engineering (to 2015)

2000

\title{
LOW COHERENCE FIBER OPTICS FOR RANDOM NOISE RADAR
}

\author{
Ruixiang Jiang \\ University of Nebraska-Lincoln \\ Kyle W. Wolfe \\ University of Nebraska-Lincoln \\ Lim Nguyen \\ University of Nebraska-Lincoln, Inguyen1@unl.edu
}

Follow this and additional works at: https://digitalcommons.unl.edu/computerelectronicfacpub

Part of the Computer Engineering Commons

Jiang, Ruixiang; Wolfe, Kyle W.; and Nguyen, Lim, "LOW COHERENCE FIBER OPTICS FOR RANDOM NOISE RADAR" (2000). Faculty Publications in Computer \& Electronics Engineering (to 2015). 67.

https://digitalcommons.unl.edu/computerelectronicfacpub/67

This Article is brought to you for free and open access by the Electrical \& Computer Engineering, Department of at DigitalCommons@University of Nebraska - Lincoln. It has been accepted for inclusion in Faculty Publications in Computer \& Electronics Engineering (to 2015) by an authorized administrator of DigitalCommons@University of Nebraska - Lincoln. 


\title{
LOW COHERENCE FIBER OPTICS FOR RANDOM NOISE RADAR
}

\author{
Ruixiang Jiang \\ Kyle W. Wolfe \\ Lim Nguyen
}

\author{
The Peter Kiewit Institute of Information Science, Technology and Engineering \\ Dept of Computer and Electronics Engineering \\ University of Nebraska-Lincoln \\ Omaha, Nebraska
}

\begin{abstract}
Coherent random noise radar has been developed at the University of Nebraska for the detection and identification of shallow subsurface objects such as land mines. Other application ranges from remote sensing, synthetic aperture radar and high-resolution interferometer, to covert target tracking. In this paper, we describe the experimental investigation of a novel application of superfluorescent fiber or erbium doped fiber amplifier (EDFA) light sources(SFS) for random noise radar. The ultra-wide band noise coupled with the use of optical fiber delay lines makes SFS ideally suited for random noise radar applications. Optical fiber makes long and multiple-step delay lines of a few kilometers feasible. Its advantage includes being extremely low-loss, compact and lightweighted, and available at a much lower cost, while avoiding the dispersion and non-linearity associated with $R F$ delay lines. We report the analysis and experimental results to characterize the resolution characteristics of the systems.
\end{abstract}

\section{INTRODUCTION}

Random noise radar employs white noise as the signal source. The transmit signal is time delayed and correlated with the received signal to obtain a correlation peak. The length of delay then determines the target range. A number of noise radar systems have been developed for moving target or landmine detection. Early in 1959, Horton [1] proposed a distance-measuring radar by transmitting modulated noise such that the distance was obtained from the correlation function. It has been suggested that range ambiguities in radar systems may be reduced by transmitting wide-band noise [2]. In [3], Dillard discussed the use of optical fiber as signal delay, matched filtering in radar signal processing. Oliver performed theoretical comparisons of radar principles at microwave and optical frequencies[4]. In the system prposed by Forrest and Meeson [5], the noise was down converted to an intermediate frequency in order to minimize the delay-line losses. The coherent noise radar was developed by Narayanan [6] to measure both the in-phase and quadrature-phase components. Coherence injection was accomplished using a local oscillator and an intermediate mixing frequency. Theron et. al. has developed an ultrawideband radar operating in the foliage penetration band [7],[8].

Traditionally, the time delay is realized with microwave delay lines that are expensive and bulky. Fiber optic delay lines have also been used but they require optical modulators and detectors to interface with the microwave signals. The electro-optic conversions have very high loss and linearity limitations.

We propose to generate the broadband microwave noise using low-coherence fiber optic light sources. SFS or EDFA light source is based on amplified spontaneous emission (ASE) that has ultra-wide bandwidth of approximately $1,200 \mathrm{GHz}$. The intense output light is readily available in optical fiber that eliminates the need for electro-optic conversions. The resolution of the radar system that employs SFS is limited only by the bandwidth of the photo-detectors. Optical fiber makes long and multiple-step delay lines of a few kilometers feasible. In our experiments, the low coherence SFS is employed in a novel electro-optical Michelson interferometer arrangement to demonstrate the feasibility of the radar application.

In this paper, we analyze the experimental system and report the results from computer simulations and measurements. We also propose a method to optically inject coherence into the radar system that employs EDFA light sources.

\section{SYSTEM DESCRIPTION}

The erbium doped fiber amplifier source is a proven source of broadband light in current applications such as fiber optic gyroscope. In our experiment, the source consisted of a $100 \mathrm{~m}$-long erbium-doped fiber that was pumped in the backward configuration by a $980 \mathrm{~nm}$ laser 
diode to generate spontaneous emissions at $1550 \mathrm{~nm}$ : An integrated fiber optic amplifier module (IFAM) from ETEK Dynamics was used to couple the pump and ASE light in and out of the fiber, respectively. The IFAM incorporates an isolator to prevent reflection back to the laser diode. The EDFA source is capable of producing $30 \mathrm{~mW}$ of optical power at $1550 \mathrm{~nm}$ when pumped by $100 \mathrm{~mW}$.

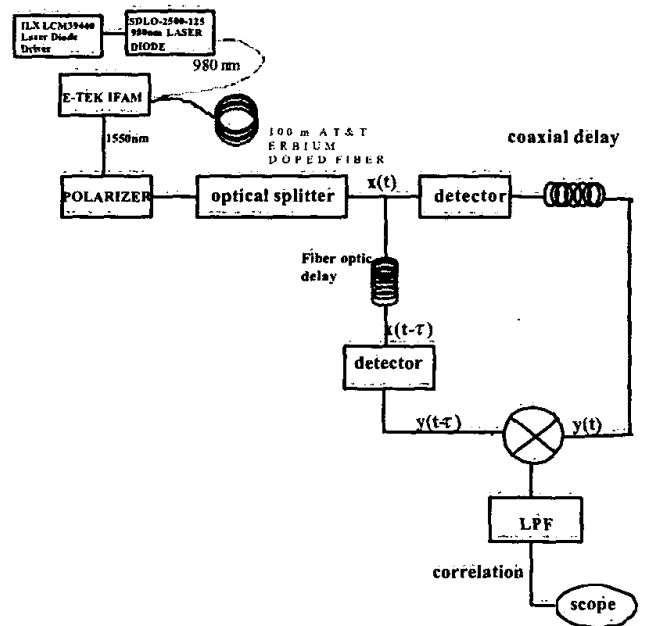

Figure 1. Block diagram of the electro-optical Michelson interferometer.

The broadband ānd unpolarized output light passed through a fiber polarizer and was coupled into an optical splitter to generate the signal and reference outputs. The photo-detector converted the signal light into the microwave noise signal that was coupled into a $50 \Omega$ coaxial cable. This coaxial delay was used to simulate the round trip time to the radar target.

The reference light propagated through an optical delay that consisted of a fiber delay line and an adjustable freespace path length. Another identical photo-detector converted the reference light into the microwave reference noise. Thus, there are two nearly identical white noise signals at the outpuit of the photo-detectors. The optically delayed reference was multiplied with the coaxially delayed signal using a wide-band mixer. The output of the mixer was low-pass filtered to yield, in terms the noise radar signals, the correlation of the received signal with the delayed version of the transmitted signal The experimental apparatus represents a Michelson interferometer in which one optical path is replaced by the RF delay and the interference is constructed in the RF domain. Thus, although the light source is ultra-wide band, the resolution of the system is limited by the RF bandwidth of the photo-detectors; which is about $1 \mathrm{GHz}$.
The correlation response was measured by adjusting the free space delay. The full-width half-max (FWHM) of the correlation response was taken to be the free-space resolution of the system, or the minimum separation at which the two targets are indiscernible. In practice, the correlation value is subjected to dispersion due to the frequency response of the radar system. The dispersion effects can be reduced by proper calibration [7].

\section{ANALYSIS}

We model the optical signals as polarized thermal light centered at some frequency $\omega_{c}$ with random amplitude and phase, $A(t)$ and $\Phi(t)$ réspectively [9]:

$$
x(t)=A(t) \cos \left[\omega_{c} t+\Phi(t)\right]
$$

The optical signals are photo-detected by two identical square-law detectors whose low-pass bandwidths, $B e$, are much smaller than the optical bandwidth, $B o$, of the spectrum of the polarized thermal light. Thus, the current outputs of the detectors are proportional to the light intensities, namely $A^{2}(t)$ and $A^{2}(t-\tau)$. The inputs to the mixer are proportional to the detector currents, $y(t)$ and $y(t-\tau)$. Here we took $\tau$ to be the total relative delay between the radar signal and the reference signal. The mixer output is low-pass filtered to give a signal that is proportional to the time average of $y(t)$ and $y(t-\tau)$, or their correlations:

$$
\Gamma(\tau)=\overline{\overline{y(t) \cdot y(t-\tau)}}
$$

Since $B e<<B o$, the power spectra of the detector currents, $Y(\omega)$, are approximately uniform:

$$
Y(\omega)=\operatorname{rect}\left(\frac{\omega}{2 B e}\right)
$$

So the normalized autocorrelation function is proportional to the inverse Fourier transform of $I(\omega)$ :

$$
\Gamma(\tau)=\operatorname{sinc}(\hat{B} e \cdot \tau)
$$

Discrete simulations were performed to analyze the experimental system. The light source was modeled using the circulär complex Gaussian band-pass process [9]:

$$
\begin{aligned}
& x_{k}[n]=\operatorname{Re}\left\{\left(R_{k}[n]+j I_{k}[n]\right) \exp (j \dot{\Omega} n)\right\} \\
& x_{k}[n]=A_{k}[n] \cos \left(\Omega n+\phi_{k}[n]\right),
\end{aligned}
$$

where $\mathrm{k}$ denotes the simulation trial. $\dot{R}_{k}, I_{k}$ are zeromean, i.i.d. Gaussian random variables: The autocorrelation function $\Gamma(m)=\frac{1}{M} \sum_{k=1}^{M} \Gamma_{k}(m)$, where $M$ is the total number of trials. $\Gamma_{k}(m)$ is the autocorrelation function for each simulation trial 
$\dot{x}_{k}[n]: \Gamma_{k}(m)=\sum_{n=1}^{N} y_{k}[n] y_{k}[n+m]$. Here $N$ is the length of $x_{k}[n] ; y_{k}[n]$ is the low-pass filtered signal of $x_{k}{ }^{2}[n] ; y_{k}[n+m]$ is the low-pass filtered signal of $x_{k}{ }^{2}[n+m]$ whose delay was computed in the frequency domain, viz, $X_{k, m}(\Omega)=\exp (j \Omega m) X_{k}(\Omega)$.

\section{RESULTS}

Figire 2 showis the example computer simulations of the system. The dc component of the low-pass signals were removed to reflect the non-dc characteristics of the New Focus photo-detectors used in the experiment. The axes in the figures have arbitrary units. The correlation response of the system is the sinc(:) function as expected.
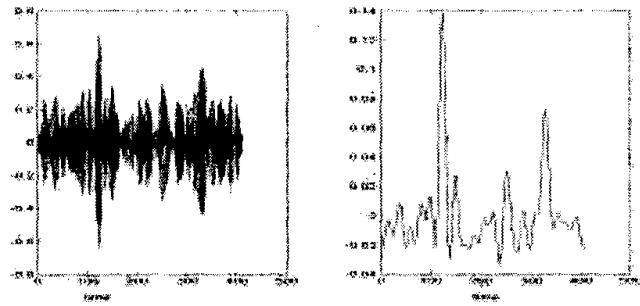

(a) EDFA light source $x(t)$, and radar signal $y(t)$.
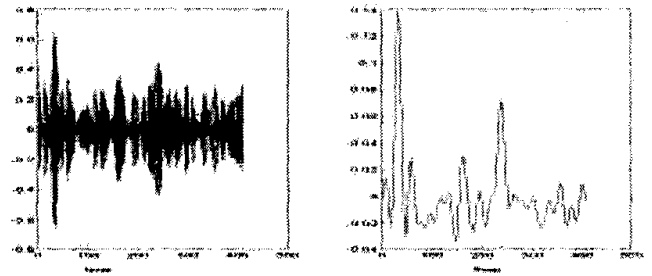

(b) Delayed optical signal $x(t-\tau)$, and reference $y(t=\tilde{\tau})$.

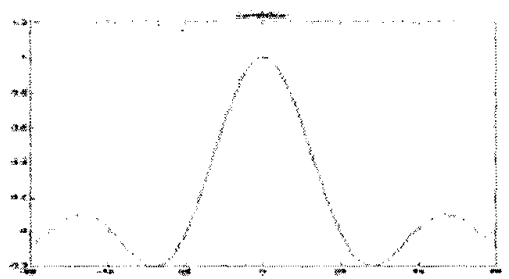

(c) Correlation output:

Figure 2. Computer simulâtion rèsults.

The system resolution, $D$, can be easily derived from the autöcorrelation function $\Gamma(\tau)$ :

$$
D=c \cdot \Delta \tau=\frac{2 c}{1.896 \cdot B e}
$$

where $\mathrm{c}$ is the speed of light and $B e$ is in radians/sec, $\Delta \tau$ is the delay at FWHM of the correlation response. For $B e=1.0 \mathrm{GHz}, D=18.11 \mathrm{~cm}$.

In the experiment, $B e$ must be corrected using the equivalent noise bandwidth of the photo-detector. The response of the photo-detector can be well approximated by a second-order Butterworth filter with a $1.0 \mathrm{GHz}$ cutoff frequency. Since the noise bandwidth factor of a second order Butterworth is 1.11 , the theoretical resolution of the system should be corrected by this factor of 1.11: $D=18.11 \mathrm{~cm} / 1.11=16.32 \mathrm{~cm}$.

Figure 3 shows the experimental results. The optical spectrum of the SFS is about $1.2 \mathrm{THz}$ at $1530 \mathrm{~nm}$ wavelength. The photo-detector output shows the broadband microwave noise that is limited by the detector bandwidth. The correlation response of the system was measured using the adjustable optical delay to yield a freespace FWHM resolution of $15.2 \mathrm{~cm}$, which is in very close agreement to the theoretical calculations.

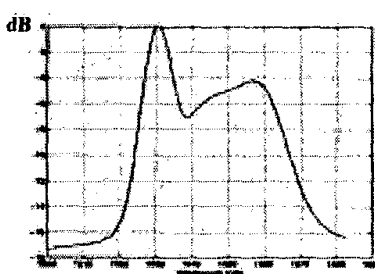

(a) optical source spectrum

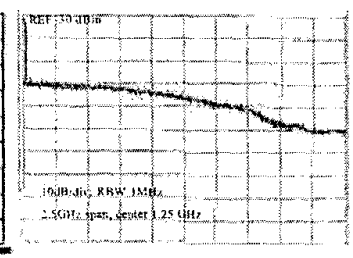

(b) RF detector spectrum

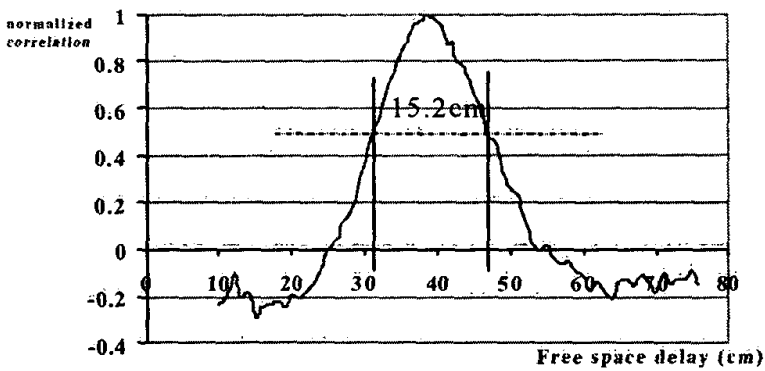

(c) Free space opticâl delay $(\mathrm{cm})$

Figure 3. Measurements of the optical spectruim, detector spectrum and the normalized correlation ressponse. 


\section{OPTICAL INJECTION OF COHERENCE}

We propose a method to optically inject coherence into the radar system that employs EDFA light sources. Coherent noise radar [6] has the advantage that the Doppler and polarimetric responses from the target could be extracted using coherence detection.

Figure 4 shows the simplified block diagram of the proposed system. The $1550 \mathrm{~nm}$ light is intensity modulated by an RF local oscillator (LO). The modulated light is photo-detected and filtered by BPF1 that is centered at $\omega_{\text {ref }}(\sim 2 \mathrm{GHz})$ with a bandwidth of $2 \mathrm{Be}$ prior to radar transmission. The received radar signal is mixed with the optically delayed signal to yield an IF signal that is fed to a narrow-band filter, BPF2, centered at $\omega_{\text {ref }}$. The amplitude of the IF signal from BPF2 is therefore proportional to the auto-correlation of the noise. The IF signal is coherently detected with the I/Q detector using the LO signal to provide the in-phase (I) and quadrature (Q) components. The amplitude and phase of the received radar signal can then be obtained from the I \& Q components, allowing the extraction of the target's polarimetric response or its Doppler shift [6].

We model the LO signal as $c(t)=\cos \left(\omega_{r e f} t+\theta\right)$.

The output of the optical modulator is:

$$
y(t)=B(t) \cos \left[\omega_{c} t+\phi(t)\right],
$$

where $B^{2}(t)=A^{2}(t)\left[1+a_{o} \cos \left[\left(\omega_{r e f} t+\theta\right)\right]\right.$, and $a_{o}$ is the modulation index.

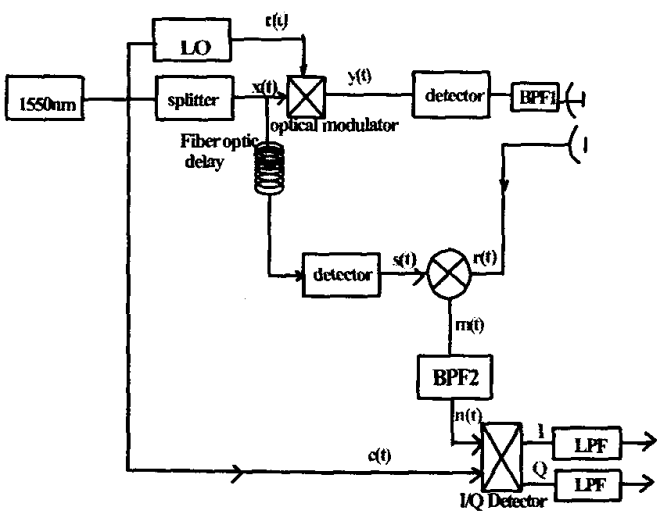

Figure 4. Coherence optical injection using EDFA.

Therefore, the bandpass filtered radar signal is proportional to $\left[A^{2}(t)+a_{o} A^{2}(t) \cos \left(\omega_{r e f} t+\theta\right)\right]^{*} h(t)$, where $h(t)$ is the impulse response of BPF1.
The optically delayed signal, $s(t)$, is proportional to $A^{2}(t-\tau) * \tilde{h}(t)$, where $\tilde{h}(t)$ is the base-band equivalence of $h(t)$. Again we took $\tau$ to be the total relative delay between the radar signal and the reference signal.

Ignoring the path attenuation, the received signal is:

$$
r(t)=f(t)+g(t)
$$

where $f(t)=a_{o}\left[A^{2}(t) \cos \left(\omega_{r e f} t+\theta+\Delta \theta\right)\right]^{*} h(t)$ is the frequency shifted version of $s(t)$, and $g(t)=A^{2}(t)^{*} h(t) . \Delta \theta$ is the relative phase introduced by the target.

The received signal, $r(t)$, and the optically delayed signal, $s(t)$, are the inputs to the mixer whose output is:

$$
m(t)=f(t) s(t)+g(t) s(t)
$$

This signal is then fed to the narrow-band BPF2. Now the noise power spectral density of the light intensity, $A^{2}(t)$, is essentially white at the RF range of interest. It follows that if $\omega_{\text {ref }}>2 B e$, then $s(t)$ and $g(t)$ are uncorrelated. So the second term in (8) does not contribute to the output of BPF2. Therefore, the amplitude of the IF signal $n(t)$, is proportional to the auto-correlation of $s(t)$ :

$$
n(t)=\Gamma(\tau) \cos \left(\omega_{\text {ref }} t+\theta+\Delta \theta\right)
$$

The in-phase and quadrature components of the IF signal are obtained via coherent detection at the $\mathrm{V} / \mathrm{Q}$ detector using the LO signal:

$$
\begin{array}{ll} 
& I=\Gamma(\tau) \cos (\Delta \theta), \\
\text { and } & Q=\Gamma(\tau) \sin (\Delta \theta)
\end{array}
$$

Figure 5 shows the example results of the computer simulation for $\Delta \theta=0$. The in-phase component, $I$, is described by a $\operatorname{sinc}($.$) function as expected.$

\section{CONCLUSION}

We have proposed and demonstrated a novel application of low coherence fiber optics for random noise radars. In our experiments, the EDFA light source was employed in a novel electro-optical Michelson interferometer arrangement to demonstrate the feasibility of the proposed approach. The experimental results have characterized the system resolution that agrees well with the theoretical analysis and computer simulations. We have also proposed a method for optical injection of a microwave carrier for coherent random noise radar applications. The principle of this scheme was verified by analysis and simulation. Plans are underway to experimentally demonstrate its operation. The low coherence fiber optic system described here will 
be incorporated into an operating random noise radar system for actual target detections and system evaluations.

\section{ACKNOWLEDGEMENT}

We would like to thank ILX Lightwave Corporation for the laser diode controller and related fiber optic equipment through their University Donation Program, and Lucent's Bell Labs for donating the erbium-doped fiber. Thanks are also due to Robert Fladby and Robert Frankland of the Computer and Electronics Engineering department. This work was partly support by the Mobil Communications Research Project of the Nebraska Research Initiatives and UNL College of Engineering and Technology.

\section{REFERENCES}

[1] B.M. Horton, "Noise-modulated distance measuring systems," Proc. IRE, vol.47, no.5, pp.821-828, May 1959.

[2] M.P. Grant, G. R. Cooper, and A.K. Kamal, "A class of noise systems," Proc, IEEE, vol. 51, no. 7, pp. 10601061, Jul 1963

[3] G. M. Dillard, "Radar signal Processing Using Fiber And Integrated Optics," Radar-77, 25-28, pp.363-367, Oct 1977.

[4] C.J. Oliver, "A comparison of radar principles at microwave and optical frequencies," Radar-77, 25-28, pp.513-516, Oct 1977.

[5] J. R. Forrest, and J. P. Meeson, "Solid State Microwave Noise Radar," Proceedings of the IEE Radar-77 Conference, pp.531-534, Savoy Place, London, Oct 25-28, 1977.

[6] R. M. Narayanan, Y.Xu, P.D. Hoffmeyer, and J. O. Curtis, "Design and performance of a polarimetric random noise radar for detection of shallow buried targets," Proceedings of SPIE Conference on Detection Technology: Mines and Mine-like Targets, vol.2496, pp.20-30, Orlando, FL, Apr 1995.

[7] I. P. Theron, E. K. Walton, and S. Gunawan, "Compact range radar cross-section measurements using a noise radar," IEEE Transactions on Antennas and Propagation, vol.46-9, pp.1285-1287, Sept 1998.

[8] I. P. Theron, E. K. Walton, S. Gunawan, and L. Cai, "Ultrawide-band noise radar in the VHF/UHF. band," IEEE Transactions on Antennas and Propagation, vol.47, no. 6, pp.1080-1084, Jun 1999.

[9] J. W. Goodman, Statistical Optics, John-Wiley, 1985.

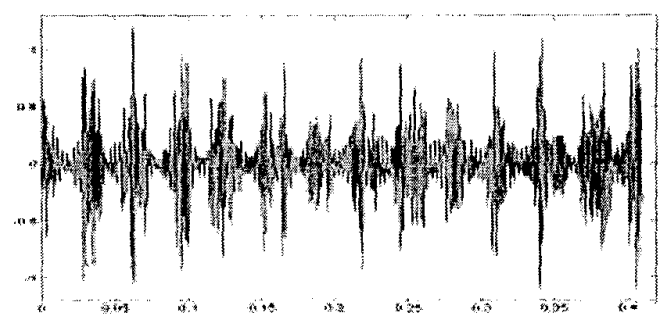

(a) Received radar signal $r(t)$.

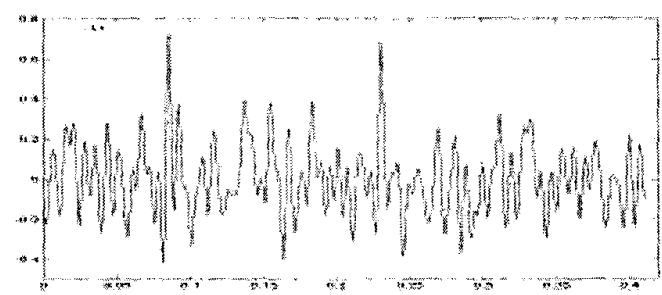

(b) Optically delayed signal $s(t)$.

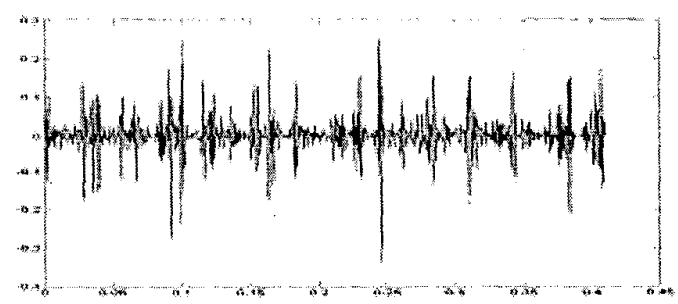

(c) Mixer's output signal $m(t)$.

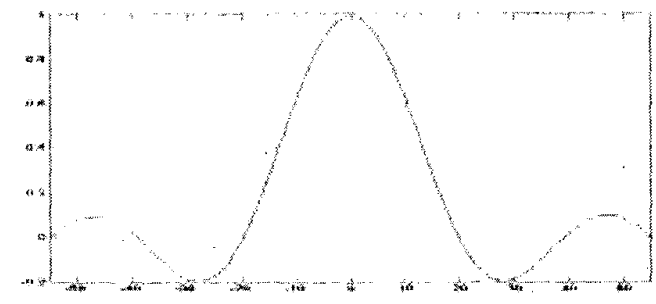

(d) In-phase signal I as a function of delay.

Figure 5. Simulation of optically injected coherence. 